\title{
Hemodiálisis domiciliaria diaria en dos pacientes
}

\author{
Elena Renau Ortells - Vicente Cerrillo García - Ma José Folch Morro - Ainhoa Agustina Trilles - Cecilia \\ Ribalta Morillón - Carmen Aicart Saura - Jesús Carratalá Chacón
}

Unidad de Diálisis. Hospital General de Castellón

\section{Introducción}

En España, en la década de los 60, eran muy pocos los hospitales que contaban con la terapia de diálisis, por la falta de espacio, recursos humanos y económicos. Esta escasez fue la que hizo que se desarrollasen programas de Hemodiálisis Domiciliaria (HDD). Hacia mediados de los años 70 se empezaron a crear más unidades de hemodiálisis y se desarrolla la diálisis peritoneal por lo que desapareció casi totalmente la HDD.

Según la Ley 41/2002 de Autonomía del Paciente, éste tiene derecho a elegir tratamiento siempre que no haya contraindicaciones médicas tras haber recibido la información oportuna. En la actualidad los profesionales damos una información más completa de los diferentes tipos de tratamientos, para que el paciente pueda elegir aquel que más se adapte a su estilo de vida. Esto, unido a la nueva concepción del paciente como centro del sistema sanitario, hace que el paciente tenga total autonomía para tomar las decisiones que conciernen a su tratamiento médico, por lo que es necesario que haya un abanico con las opciones que el paciente pueda elegir.

Recientemente se ha incorporado al arsenal terapéutico la hemodiálisis corta diaria, que es aquella que se realiza de 2 a 2,5 horas al día durante 6-7 días a la semana. Permite un perfil de concentración de solutos mucho más regular, con menores concentraciones pre-hemodiálisis de urea, creatinina, potasio e hidrogeniones. Además, es más suave y gradual, lo que hace que haya mayor estabilidad cardiovascular, por lo que sería la técnica ideal para realizarla en

\begin{tabular}{|c|}
\hline Correspondencia: \\
Elena Renau Ortells \\
Unidad de Diálisis \\
Hospital General de Castellón \\
Avda. Benicasim s/n \\
12004 Castellón \\
elerenau@hotmail.com \\
\hline
\end{tabular}

el propio domicilio del paciente, gracias también a que contamos con nuevos avances tecnológicos. Numerosos estudios señalan que con esta forma de tratamiento se produce una mejoría en la calidad de vida, control de la tensión arterial, del metabolismo fósforo- calcio, una mejoría en el patrón del sueño, así como la regresión de la hipertrofia ventricular y disminución de los episodios de hospitalización. Aunque hay datos que avalan una mejoría en la supervivencia de los pacientes, todavía son necesarios estudios que confirmen estas hipótesis.

Con todos estos antecedentes, hemos puesto en marcha en nuestro hospital un programa de entrenamiento para aquellos pacientes que eligiesen esta técnica de hemodiálisis corta diaria en domicilio.

Presentamos dos casos clínicos con el fin de mostrar nuestra experiencia con la hemodiálisis diaria domiciliaria en los dos primeros pacientes entrenados para esta técnica.

Para llevar a cabo el programa de Hemodiálisis Domiciliaria Diaria (HDDD), es necesaria una infraestructura adecuada. En nuestra unidad se habilitó una sala para el entrenamiento de los pacientes que pudiesen elegir esta opción. Aparte del soporte material, se seleccionó a una enfermera responsable de la formación y entrenamiento de estos pacientes.

Para la inclusión de estos pacientes tuvimos en cuenta que el paciente tuviera:

- Una situación biológica adecuada.

- Una cierta capacidad de aprendizaje.

- Motivación para realizar la técnica en su domicilio.

- Buen acceso vascular.

- Una persona de ayuda

- Un espacio y condiciones higiénico - sanitarias adecuadas. 
El paciente y/o familiar debían conocer muy bien el manejo de la técnica, sus complicaciones y como solventarlas. Por ello, la formación que reciban estos pacientes antes de comenzar el tratamiento en su domicilio, es de suma importancia.

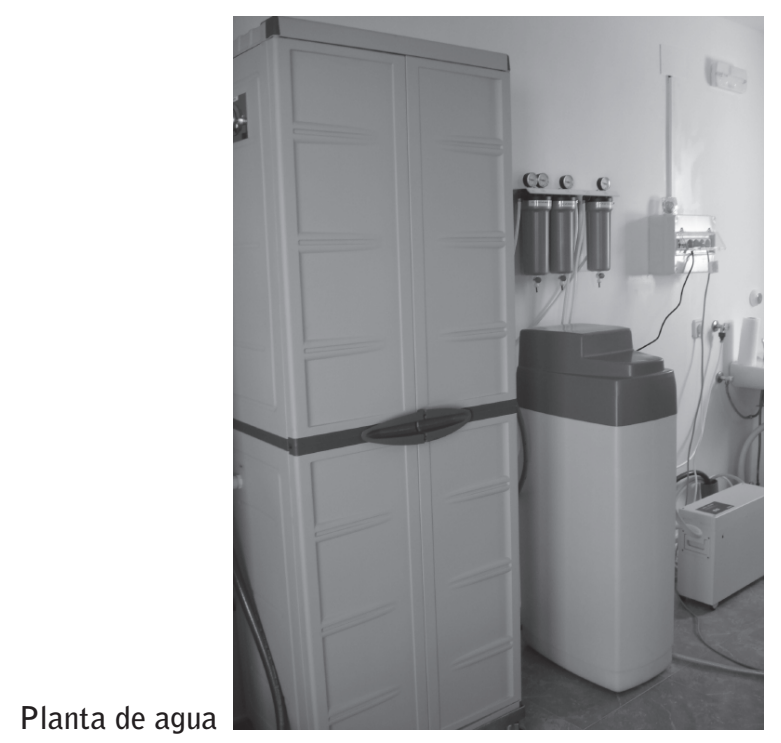

\section{DESCRIPCIÓN DE LOS CASOS}

\section{Caso 1.}

Paciente de 50 años que era tratado en consulta de Nefrología desde mayo del 2001 por una insuficiencia renal secundaria a poliquistosis, presentando hipertensión, dislipemia, hiperuricemia y síndrome prostático. Empezó la diálisis de forma urgente el 26/10/07 con un catéter temporal. Se le implantó catéter tunelizado y se le realizó la fístula arteriovenosa interna radial izquierda sin éxito, por lo que se hizo un nuevo intento en la arteria humeral izquierda, que tampoco funcionó, por lo que se decidió dejar descansar al paciente y empezar el entrenamiento con el catéter tunelizado.

La toma de contacto del paciente con enfermería fue cuando lo ingresaron por deterioro de la función renal, ya que el nefrólogo lo derivó a la consulta de Enfermedad Renal Crónica Avanzada, para informarle de las diferentes opciones de tratamiento renal.

En la recogida de datos se observó que el paciente estaba trabajando en una pastelería, tenía capacidad para aprender y disponía del apoyo de su mujer. Al explicar las diferentes modalidades, ventajas y desventajas de cada una de ella, le ofertamos la HDDD, pero el paciente pareció inclinarse por la DPCA. Le dejamos un tiempo para que asimilara la información, le dimos material didáctico y le informamos de algunas páginas de Internet que podía leer. Tras el fin de semana de reflexión, el paciente y su esposa decidieron que la técnica que menos alteraría su estilo de vida era la HDDD; ya que en esta técnica solo invertirían 2 horas, en su propio domicilio y sin un horario estricto.

Se empezaron a programar las sesiones educativas a las que acudían el paciente y su esposa. Para ello, hicimos un protocolo de entrenamiento, en el que constaba en cada semana los temas a tratar, sin pasar al tema siguiente si el de la semana anterior no se sabía. Empezamos por conocimientos básicos como significado de insuficiencia renal, fenómeno de diálisis, alimentación adecuada. Continuamos con la importancia de un control diario del agua: cloro, cloraminas y dureza. Posteriormente, pasamos a las partes del monitor, preparación, montaje, posibles alarmas. Mas tarde, seguimos con la conexión del paciente, que, como anteriormente se ha mencionado, era portador de catéter tunelizado, por lo que explicamos la importancia de una técnica estéril. Mientras el paciente estaba conectado, aprovechábamos para explicar las alarmas, los síntomas indeseables más frecuente durante la diálisis, y la forma de minimizarlos una vez estuviera en su casa. Por último, tratamos el tema de la desinfección del monitor.

Mientras tanto, en casa, se fue instalando la planta de agua, y fueron el técnico y la enfermera de la casa comercial que proporcionó el sistema quienes se encargaron de explicar su funcionamiento. Una vez instalado éste, se hizo un control de análisis químico y bacteriológico del agua que demostró la presencia de $1000 \mathrm{ufc} / \mathrm{ml}$ de gérmenes aeróbios mesófilos, por lo que se aumentó la capacidad decloradora.

También se instaló una cámara de alta resolución para mantener un contacto directo a través de videoconferencia con el paciente, lo que nos permite visualizar el orificio del catéter, la forma de conexión o cualquier otra cuestión que se plantee.

\section{Caso 2.}

Paciente varón de 82 años tratado en la consulta de Nefrología desde enero del 2007 por una insuficiencia renal secundaria a nefroangiosclerosis, presentaba hipertensión y diabetes tipo2. Se le realizó fístula radiocefálica izquierda sin éxito por lo que se decidió poner catéter tunelizado en vena yugular derecha y empezar la diálisis de forma programada. Posteriormente, se realizó otra fístula humeral, que tampoco funcionó. 


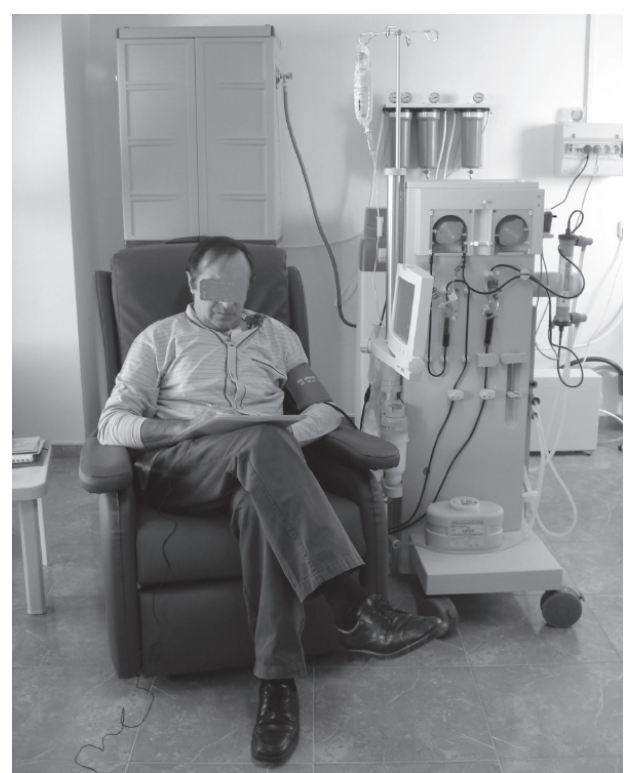

Paciente conectado a monitor

El paciente, a pesar de la edad, se mantenía activo en su empresa de análisis clínicos, a la que acudía a diario, por lo que las horas invertidas en el hospital haciendo la diálisis le suponían un gran impedimento para el trabajo. En la sala el paciente se mostraba muy nervioso así que le informamos de la existencia del programa de HDDD, pareció gustarle ya que el día siguiente vino un hijo a pedirnos información y se decidió iniciar el entrenamiento. En principio, seguimos el mismo procedimiento que con el anterior paciente pero vimos que este paciente no sería capaz de seguirlo, por lo que se propuso que algún familiar aprendiera. Decidieron que se entrenará su hijo y una enfermera.

Empezamos a programar las sesiones educativas, y comprobamos que para cada paciente hay un ritmo de aprendizaje, por lo que tuvimos que amoldar los protocolos anteriores. Estos nos sirvieron de guía o recordatorio y el periodo de entrenamiento fue un poco mas largo en este caso, ya que las dos personas que venían a aprender no venían juntas, sino en días alternos. No hubo ningún problema con la adecuación del domicilio ni con la instalación de la planta de agua.

La duración de ambos entrenamientos fue de 2 meses y 2,5 meses, respectivamente. Para el entrenamiento, aprovechamos las tres sesiones semanales de diálisis pautadas con una duración de cuatro horas. El material que utilizamos para el entrenamiento fue el mismo que llevarían los pacientes en casa: el filtro empleado en los dos casos polisulfona de 1.7 (Xenium $170 \circledR)$, el líquido de diálisis con calcio 30 y potasio de 2. Se instaló en el domicilio un monitor modelo
Aurora de Baxter ${ }^{\circledR}$, equipado con tensiómetro, pantalla táctil y regulable en altura. La velocidad de la bomba se programó a $350 \mathrm{ml} / \mathrm{min}$ y la velocidad del líquido de baño a $800 \mathrm{ml} / \mathrm{min}$. Se pautó para el domicilio una frecuencia de tratamiento de 6 días por semana y 2 horas de duración de la sesión.

En ambos casos cuando la formación se completó fuimos al domicilio para dar el visto bueno a la instalación, y hacer la primera diálisis con la compañía y apoyo de nuestro equipo (nefrólogo, enfermera y técnico). No se plantearon dificultades importantes, salvo el momento de la conexión del catéter, debido a que no estaban familiarizados con la colocación de guantes y lo que implicaba una técnica estéril.

Para evitar problemas en casa a los pacientes les proporcionamos el teléfono de la unidad y les aconsejamos que su horario de tratamiento estuviera comprendido entre las 8 y las 22 horas, por si apareciera cualquier problema pudiéramos solucionarlo.

Los dos pacientes se fueron a casa con catéter permanente y a espera de realizarse nueva fístula ya que, aunque teóricamente la conexión al catéter es más sencilla para los pacientes, hay que intentar evitar éste, por el mayor riesgo de infecciones que supone.

Como en la experiencia de otros centros, hemos aconsejado que el paciente no se halle sólo en su domicilio en el momento de la HD y es muy importante que el paciente se sienta apoyado en su domicilio tanto por un familiar como con nuestro equipo. La hemodiálisis domiciliaria debe considerarse como una opción reversible, y el paciente y su familia deben entender que la posibilidad de volver a dializarse en el hospital o en un centro siempre queda abierta.

Muchos estudios avalan los beneficios de la hemodiálisis diaria, pero que comporta grandes dificultades de organización en las unidades, por lo que la hemodiálisis domiciliaria diaria, sería una buena alternativa y una excelente opción de tratamiento para pacientes estables en el futuro. Aunque existen numerosos estudios sobre hemodiálisis domiciliaria, son escasa las experiencias con en HDDD siendo esta una modalidad terapéutica útil y susceptible de ser realizada por cualquier servicio de Nefrología, siempre que disponga de una infraestructura adecuada. En nuestro caso todos los problemas han sido resueltos por teléfono, sin necesidad de desplazamientos.

Debido a nuestra corta experiencia, no tenemos suficientes datos que nos confirmen una mejoría en las 
analíticas de estos dos pacientes, pero estos afirman que se encuentran mucho mejor y que disponen del tiempo necesario para realizar sus actividades sin restricciones horarias y por tanto, que tienen una mejor calidad de vida.

Actualmente, estamos entrenando a una mujer de 55 años derivada de la consulta de diálisis peritoneal, que vive a una distancia de nuestro centro de $100 \mathrm{Km}$ y trabaja en una granja agrícola y pensamos que si informamos a los pacientes de las diferentes opciones de tratamiento desde las consultas de enfermería de Enfermedad Renal Crónica Avanzada, los pacientes que están activos laboralmente pueden tender a inclinarse por las terapias domiciliarias.

\section{Bibliografía}

1. Barahona Muñoz MT, Tabares Galán M. Hemodiálisis domiciliaria una modalidad terapéutica olvidada. Análisis retrospectivo de 20 años de experiencia. Servicio Nefrología Hospital Ramón y Cajal (Madrid). En: Libro de comunicaciones del XXIV Congreso de la SEDEN; Valencia 2023 octubre 1999. Barcelona: HOSPAL; 1999. p. 48-54.

2. Barril G, Sánchez Tomero JA. La hemodiálisis domiciliaria alternativa posible y necesaria. Nefrología 2007; 27(1):3-4.

3. González A, Julián JC. Hemodiálisis domiciliaria. Revista Alcer 2007; julio-septiembre $n^{\circ}$ 142:1824. 